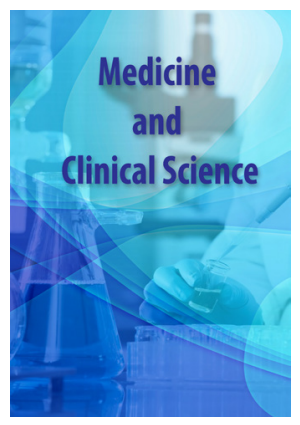

Correspondence

Edward W. Braun

Midtown Medical Center \& Spa, 919 N. Dale Mabry Hwy Suite 300, Tampa, FL 33614, USA

Tel: 813-935-3221

Fax: 813-933-8149

E-mail:Dr.Braun@DrEdwardBraun.com

- Received Date: 04 Nov 2019;

- Accepted Date: 07 Dec 2019;

- Publication Date: 17 Dec 2019.

Copyright

(C) 2020 Science Excel. This is an openaccess article distributed under the term of the Creative Commons Attribution 4.0 International license.

\title{
Monitoring Viral Suppression when switching from fixed dose combination efavirenz, emtricitabine and tenofovir (Atripla) to fixed dose combination elvitegravir, cobicistat, emtricitabine, and tenofovir (Stribild) in virologically suppressed adults with HIV
}

\author{
Edward W Braun \\ Midtown Medical Center, Tampa, Florida, USA
}

\begin{abstract}
A Phase IV, prospective, observational cohort study examining patients changed from Atripla to Stribild for continued suppression of viral load, effect on CD-4 cells, quality of life and sleep using blood tests and standardized questionnaires. While continuing to control the virus to acceptable levels, CD 4 counts were noted to rise and patients experienced fewer CNS side effects. Sleep quality was also improved.
\end{abstract}

\section{Introduction}

Efavirenz has been part of the treatment of Human Immunodeficiency Virus (HIV) or Acquired Immunodeficiency Syndrome (AIDS) for many years including its inclusion as part of the first single tablet regimen comprising a complete Highly Active Anti-Retroviral Therapy (HAART) regimen. Because of its long term clinical data and effectiveness as an antiviral agent, it has been one of the most popular medications used in the treatment of HAART regimens for many years. It has been widely used despite well-known side effects associated with the medication. The most common side effects associated with the medication include hypercholesterolemia, sleep disturbances, dreams, and cognitive impairment. But, the risk / benefit balance has weighed in favor of use of the medication because of its efficacy, lack of better options, and unique option of being part of the only single tablet regimen for many years. Due to its efficacy and convenience, it was part of the recommended treatment regimen for naïve patients for many years.

However, recent changes to the US Department of Health and Human Services (DHHS) HIV treatment guidelines [1] have downgraded efavirenz from one of the preferred regimens to an "alternate" regimen for the treatment of HIV naïve patients. Efavirenz is becoming less popular as part of HIV treatment regimens because of the development of newer options that may be even more effective at suppressing HIV viral loads and have fewer central nervous system (CNS) side effects. Also, the development and approval of other single tablet regimens to treat HIV positive patients have increased the number of options available with the simplicity of one tablet HAART regimens.
Recent studies have shown the CNS toxicity associated with efavirenz can be improved by changing to Raltegravir [2]. And other studies have shown that a newer regimen of coformulated elvitegravir, cobicistat, emtricitabine, and tenofovir (Stribild) is non-inferior to fixed dose combination efavirenz, emtricitabine and tenofovir (Atripla) with regard to maintaining viral suppression [3] The CNS side effects associated with Atripla may be lessened or alleviated by changing to newer single tablet regimens that do not include efavirenz. As such, Stribild might be a more favorable medication that can provide continued suppression of the HIV virus with fewer CNS side effects and improved sleep quality resulting in better quality of life for patients.

Since many patients have been taking Atripla for a long time, they may have adapted to the inherent side effects associated with efavirenz as their "new normal". The patients may be accommodating to the sleep disturbances and cognitive impairments unnecessarily. If this is true, these patients may benefit from a change to newer HAART options with fewer side effects that could provide a better quality of life.

Therefore, the study was designed to evaluate how patients faired when changing from Atripla to Stribild while monitoring changes in viral load, T-cell count, reported CNS impairment, sleep quality, and quality of life.

\section{Methods}

Patients were selected who were taking fixed dose combination efavirenz, emtricitabine and tenofovir (Atripla) as their first and only medication for their HIV treatment. The patients had to take Atripla for at least 3 months with suppressed viral loads $(<50$ copies $/ \mathrm{ml}$ ) at the time of the screening visit. The 
inclusion criteria also included age $>18$ years old and a recent estimated Glomerular Filtration Rate (eGFR) $>70 \mathrm{~mL} / \mathrm{min} / 1.73 \mathrm{~m}^{2}$ based on Modification of Diet in Renal Disease (MDRD) Study calculation.

Patients were excluded if they were pregnant or refused to use contraception during the time of the study. Patients were also excluded if they were participating in another study at the same time.

Patients were screened and identified to be potential candidates for the study by searching our database for patients diagnosed with HIV or AIDS and currently taking Atripla. The office manages approximately three hundred (300) HIV+ patients. A search of the patient database found about seventy-five (75) patients who had a history of ever taking Atripla (past or present). Further review of the medical records found approximately forty (40) patients who met the study criteria; ie (i) Atripla was the first medication that had been prescribed, (ii) taking Atripla at the time of screening, (iii) had been taking the medication for at least three (3) months, and (iv) had viral load $<50$ while taking Atripla. These patients were called and asked to visit the office or screened during their regularly scheduled office visit. Twenty-eight (28) patients expressed interest in participating. They were counselled regarding the study and were required to give informed consent. During screening / baseline visit, blood was collected for viral load, CD-4 cell count, and basic metabolic panel to evaluate for study eligibility. Patients were then given the St Stephens Aids Trust 047 Questionnaire [4] (SSAT) to evaluate for CNS toxicity and the Pittsburg Sleep Quality Index (PSQI) [5] to evaluate quality of their sleep.

The SSAT 047 scores 10 items related to efavirenz side effects including: dizziness, depression, insomnia, anxiety, confusion, impaired concentration, headache, somnolence, aggressive mood and abnormal dreams. The side effects were scored as 0 for "None", 1 for "Mild", 2 for "Moderate" and 3 for "Severe". Therefore a lower score reflected less impairment and a higher score represents more dysfunction.

The Pittsburgh Sleep Quality Index (PSQI) is used to measure the quality and patterns of sleep in adults. It rates sleep based on seven domains: subjective sleep quality, sleep latency, sleep duration, habitual sleep efficiency, sleep disturbances, use of sleep medication, and daytime dysfunction over the last month. It is a self-administered questionnaire covering these seven areas of sleep. Scoring of the answers is based on a 0 to 3 scale, whereby 3 reflects the negative extreme on the Likert Scale. The PSQI has been validated in several clinical studies [6-8].

\begin{tabular}{|l|c|}
\hline Patient Demographics & $\mathbf{N}=\mathbf{2 1}$ * \\
\hline Male, $\%$ & 95 \\
\hline Mean age, years (range) & $48.5(34-67)$ \\
\hline Race, $\%$ & \\
\hline Caucasian & 76 \\
\hline Black & 19 \\
\hline Native American & 5 \\
\hline Ethnicity, \% & \\
\hline Hispanic / Latino & 10 \\
\hline Viral Load (range), copies/ml & 0 (undetectable - 22) \\
\hline$<50, \%$ & 100 \\
\hline$<20, \%$ & 95 \\
\hline $\begin{array}{l}\text { Mean CD4+ count, cells/ml } \\
\text { (range) }\end{array}$ & $726(368-1797)$ \\
\hline$<200$ cells/ml, \% & 0 \\
\hline *Tof of 8 patients scrients & \\
\hline
\end{tabular}

*Total of 28 patients screened with 7 screen failures. 21 patients started and completed the study.

Table 1. Patient Demographics.
Follow up visits were conducted at 4, 12 and 24 weeks +/- 7 days for each scheduled visit. Respective visits included testing as follows:

Week 4: Complete Blood Count (CBC), Comprehensive Metabolic Panel (CMP), HIV Viral Load (VL), SSAT and PSQI scores (CD4 count not performed as this was not considered to be consistent with normal standard of care at a 4 week follow up visit)

\section{Week 12: CBC, CMP, CD4 count, VL, SSAT and PSQI scores}

\section{Week 24: CBC, CMP, CD4 count, VL, SSAT and PSQI scores}

The primary end point was the percentage of subjects who maintained HIV-1 viral loads $<50$ copies $/ \mathrm{ml}$ following the switch. Secondary end points included: changes in CD4 count, changes in SSAT Questionnaire score and changes on PSQI scores.

Data for the SSAT and PSQI was collected for each visit and changes over the course of the study were evaluated for evidence of improvement or deterioration. The answers to each questionnaire were given a numeric value based on the interpretative instructions of each respective instrument. The changes of these values were then calculated to determine change in the patient's condition. The patient demographics are represented in Table 1.

A total of 28 patients were screened for the study. A total of seven (7) patients failed their screening visit or were unable to complete the study. Three (3) patients were disqualified due to elevated viral load at the screening visit $(>50)$. Two other patients withdrew consent as they moved out of the area and were not able to return for visits after the initial screening visit. One patient was not able to obtain the medication through his insurance company. And one patient developed a rash after starting the new medication causing discontinuation of Stribild. (The rash was subsequently diagnosed as due to an unrelated medical condition.)

\section{Results}

After 24 weeks, $95 \%$ of the switched patients continued to have viral suppression $<50$ copies $/ \mathrm{ml}$. All values throughout the study were $<50$ copies $/ \mathrm{ml}$ with the exception of two patients. One experienced a viral "blip" at week 12 with a value of 154 . However, without any change in therapy, this value returned to undetectable when rechecked at week 24. A different patient had a level of 61 at week 24 (Graph 1) Neither of these low levels of detectable viremia caused any change in the treatment of the respective patients.

Likewise, there was an increase in CD 4 cells from baseline to 24 weeks of 81 cells (from 691 to 772 ).

There was a slight rise in creatinine from initial value of $0.99 \mathrm{mg} / \mathrm{dl}$ to $1.12 \mathrm{mg} / \mathrm{dl}$. (Graph 3) There were no discontinuations in the medication due to side effects or need to alter therapy due to untoward effects of the new medication.

The sum of the seven responses is reported as the total PSQI score. The higher the score, the poorer the sleep and decreases in the PSQI score indicate improved sleep quality. Over the course of our study, there was a decrease in the PSQI score from 7.3 to 5.1 correlating to improved sleep quality.

There was a large decrease in the average CNS Toxicity Score as measured by the SSAT 047 scale (Graph 5). This started at the first evaluation period of week \#4. The change seemed to reach maximum at Week 12 , then remained steady throughout the remainder of the study to week 24. Graph 6 shows the ten (10) individual components of the SSAT test and how the components changed over time. A few of the components showed minimal change or no effect with the change of the medication, including: dizziness, confusion and headache. However, a majority of the components showed significant improvement over the course of the study including: depression, insomnia, anxiety, impaired concentration, somnolence, aggressive mood and abnormal dreams. Most of these components showed improvement at the first measurement interval at week 4 after making the medication change. 


\section{Viral Load}

300

250

200

150

100

50

o
Baseline
Week 4

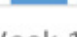

Week 24

Graph 1.Viral load remained $<50$ copies/ml during the study period.

\section{4 Cells}

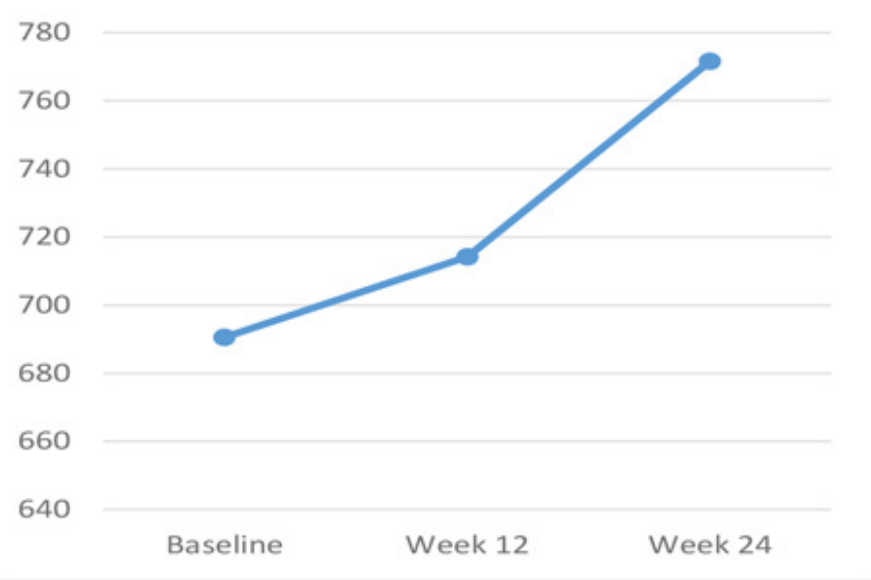

Graph 2. CD 4 cell count showed increase over the study period.

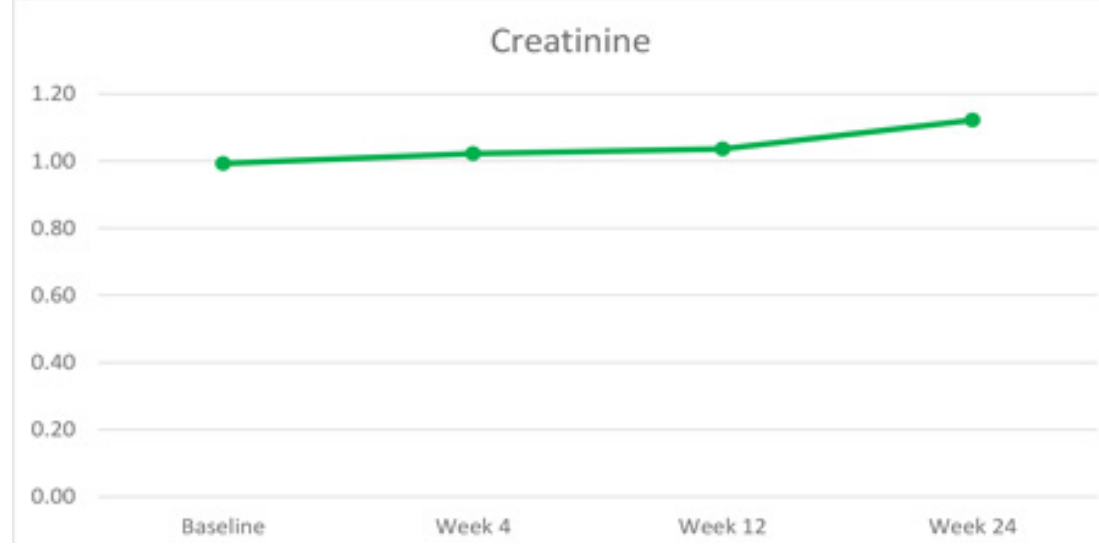

Graph 3. Creatinine measurements

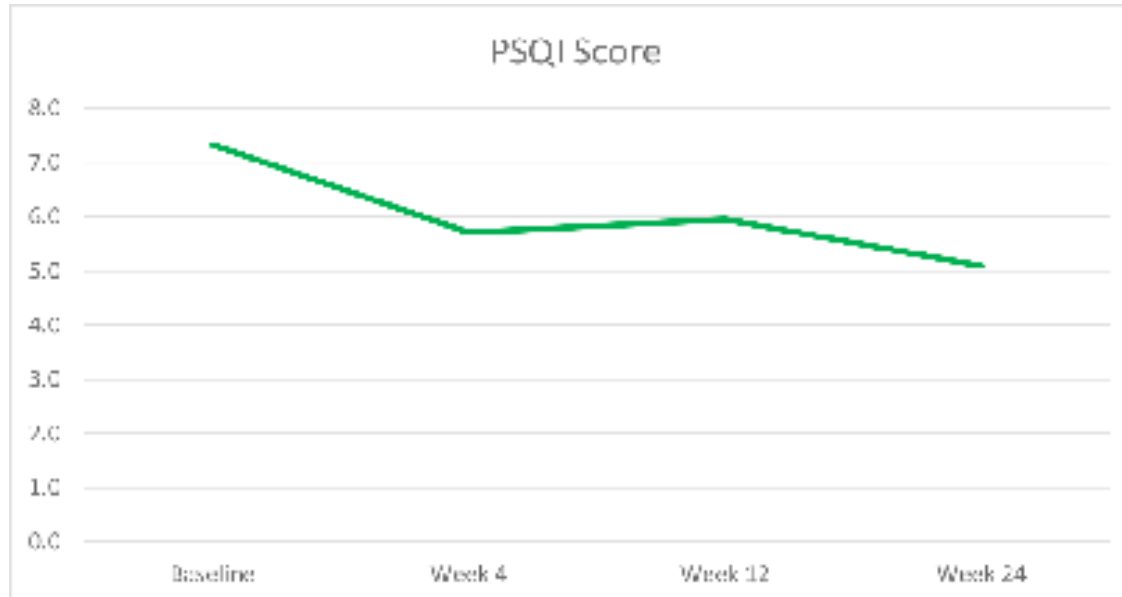

Graph 4. Creatinine measurements 


\section{Average CN5 Toxicity Score}

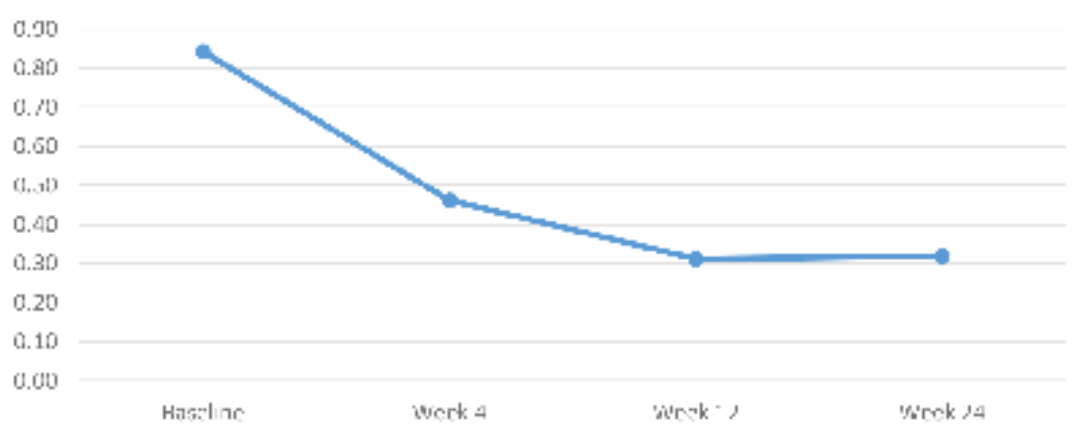

Graph 5. Decline of CNS toxicity demonstrated by the average of the SSAT 047 scale

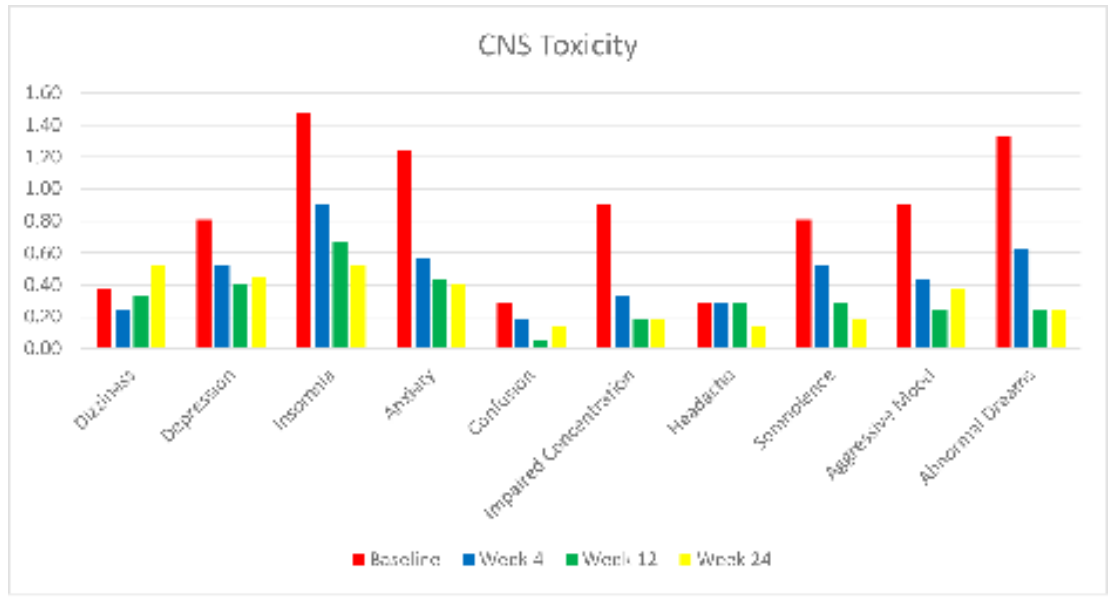

Graph 6. The 10 individual measures of the SSAT047 scale over the term of the study.

\section{Discussion}

Although Atripla has been a very important part of the armamentarium to treat HIV, we now have better choices that can achieve the same efficacy with fewer side effects [3]. Many patients who are taking Atripla could benefit from being changed to a newer regimen with the same single tablet regimen simplicity for compliance but with fewer side effects. Patients that have been taking Atripla for a long time have become accustomed to the side effects of the medication. The patients have had to adapt to the CNS complications in order to survive and keep their HIV suppressed. However, these patients now have an option available that can provide them the same sustained suppression of their virus without many of the CNS side effects that have become part of their life.

This study suggests that these patients may have improvement of their sleep quality and quality of life by changing their HAART regimen to one of the newer options which eliminates efavirenz. It is important to note that this study has limitations due to the design and size of the study. It was an open label study and patients were aware of the study design that could influence their subjective reporting of symptoms. Likewise, there was a small number of participants in the study $(\mathrm{N}=21)$ which limits the conclusions that can be drawn from the study. Also, the study was a single arm study that did not have a comparative arm.

Therefore, it is impossible to draw any conclusions from the data. However, the trend would suggest there were improvements in the secondary endpoints that were measured; sleep quality and quality of life. While the switch did not cause any loss of viral control, $95 \%$ of the participants continued to have viral loads less than 50 at the 24 week visit. Also, the one patient that did have a viral load $>50$ did not have his regimen changed due to the "blip" in his viral load.

Additional studies could be conducted to further investigate these theories to include larger, blinded studies that can demonstrate the benefit of updating HAART regimens that are effectively treating HIV infections but may be having some unwanted side effects. These changes could result in improved quality of life for these HIV positive patients. We have passed an age in HIV therapy when simply obtaining HIV suppression is sufficient. Health care providers now have the responsibility to provide updated alternatives that provide excellent potential for viral suppression with fewer potential side effects.

\section{Study Design Approval}

Study was submitted to and approved by Western Institutional Review Board ${ }^{\star} 1019$ 39th Avenue SE Suite 120 | Puyallup, WA 98374-2115, USA

\section{Funding}

Gilead Sciences, Inc.

ClinicalTrials.gov Identifier: NCT02477527

\section{References}

1. Panel on Antiretroviral Guidelines for Adults and Adolescents. Guidelines for the use of antiretroviral agents in HIV-1-infected adults and adolescents. Department of Health and Human Services. Accessed June 14, 2015. Page F-3, table 6. Available at http://www.aidsinfo.nih. gov/ContentFiles/AdultandAdolescentGL.pdf 
2. Mascolini M, Quick CNS Improvement After Switch from Efavirenz to Raltegravir, IAS 2013: 7th IAS Conference on HIV Pathogenesis, Treatment and Prevention, June 30-July 3, Kuala, Lumpur, Malaysia, http://www.natap.org/2013/IAS/IAS_39.htm

3. Pozniak A, Markowitz M, Mills A, et al. Switching to coformulated elvitegravir, cobicistat, emtricitabine, and tenofovir versus continuation of non-nucleoside reverse transciptase inhibitor with emtricitabine and tenofovir in virologically suppressed adults with HIV

4. (STRATEGY-NNRTI): 48 week results of a randomized, open-label, phase 3b non-inferiority trial. The Lancet 2014; 14: 590-599.

5. A Pilot Study to Assess the Feasibility of Switching, Individuals Receiving Atripla With Continuing Central Nervous System (CNS) Toxicity, to a Fixed Dose Combination of Tenofovir/Emtricitabine/Rilpivirine", St Stephens Aids Trust; https://clinicaltrials.gov/ct2/show/NCT01701882
6. Instruments: Pittsburgh Sleep Quality Index (PSQI)". University of Pittsburgh Sleep Medicine Institute. University of Pittsburgh. Retrieved 28 August 2015.

7. Buysse DJ, Reynolds CF, Monk TH, et al."The Pittsburgh sleep quality index: A new instrument for psychiatric practice and research". Psychiatry Research 1989; 28(2): 193-213.

8. Grandner MA, Kripke DF, Yoon IY, et al. "Criterion validity of the Pittsburgh Sleep Quality Index: Investigation in a non-clinical sample.". Sleep and biological rhythms. 2006; 4(2): 129-139.

9. Mollayeva T, Thurairajah P, Burton K, et al. The Pittsburgh sleep quality index as a screening tool for sleep dysfunction in clinical and non-clinical samples: A systematic review and meta-analysis. Sleep medicine reviews. 2016; 25:52-73. 